\title{
Isolation and Morphological Characterization of Monilinia sp. KV-27 Associated with Apple Anthracnose of Fuji Apples in Korea
}

\author{
Vivek K. Bajpai ${ }^{1}$, Jung In Yoon ${ }^{1}$, Seak Won Choi ${ }^{2}$ and Sun Chul Kang ${ }^{1 *}$ \\ ${ }^{1}$ Department of Biotechnology, Daegu University, Kyoungsan 712-714, Korea \\ ${ }^{2} B$ \& L Agro Co. Ltd., Andong, Kyoungbook 760-300, Korea \\ (Received on September 21, 2009; Accepted on October 18, 2009)
}

This study was undertaken to isolate and to identify a fungal pathogen Monilinia sp. KV-27 associated with apple anthracnose. Rotted Fuji apples were used for the isolation of the fungus. The infected tissues were sterilized with $70 \%$ ethanol, washed with sterilized distilled water and were transferred to $50 \mathrm{ml}$ containing potato dextrose broth (PDB) flasks. The peripheral hyphae of the fungal colony which developed from the infected tissues were isolated on to potato dextrose agar (PDA). On PDA plates the fungus grew well at $25^{\circ} \mathrm{C}$ and occupied more than half of a $9 \mathrm{~cm}$ petri dish within 5 days. The fungal cultures on PDA were used for morphological observation and identification of the fungus. Conidiophores were produced on the gray to whitish sporodochial structures scattered on PDA plates. These conidiophores gave rise to chains of conidia, which were branched and easily detached in water. These structures were dark brown to black and consisted of hyphal masses. Conidia produced on PDA plates were hyline or light colored, lemon shaped or ellipsoidal (10-13 $\times$ 8.5-11 $\mu \mathrm{m})$ in size.

Keywords : anthrocnose, Fuji apple, Monilinia sp. KV-27, morphological identification

Apple anthracnose is an important disease hampering the quality and texture of apple fruits worldwide. This disease is caused by Monilinia spp., which can not be distinguished solely by symptoms and signs on infected tissues. Their identification is based on morphological characters. They are comprised of living filaments known as hyphae, or collectively as mycelium. They reproduce by spores which may be sexual or asexual. Fungi are major causes of plant disease, accounting for perhaps $70 \%$ of all the major crop diseases (IAPSC, 1985). Some of these fungal plant pathogens are termed biotrophic because they establish an intricate feeding relationship with living host cells. Others are termed necrotrophic, because they invade the plant tissues

\footnotetext{
*Corresponding author.

Phone) +82-53-850-6553, FAX) +82-53-850-6559

E-mail) sckangj@daegu.ac.kr
}

aggressively, killing the host cells to obtain nutrients (Deacon, 1997).

The apple is the pomaceous fruit of the apple tree, species Malus domestica in the rose family Rosaceae which has potential nutritive and therapeutic values. Research suggests that apples may reduce the risk of colon cancer, prostate cancer and lung cancer (NRCR, 2008). Compared to many other fruits and vegetables, apples contain relatively low amounts of Vitamin $\mathrm{C}$ as well as several other antioxidant compounds (Boyer and Liu, 2004). The fiber content, while less than in most other fruits, helps regulate bowel movements and may thus reduce the risk of colon cancer. They may also help with heart disease, weight loss, and controlling cholesterol, as they do not have any cholesterol, have fiber, which reduces cholesterol by preventing re-absorption, and are bulky for their caloric content like most fruits and vegetables (AKYFH, 2008; Sharma, 2005). There is evidence that in vitro apples possess phenolic compounds which may be cancer-protective and demonstrate antioxidant activity (Lee, 2004). The predominant phenolic phytochemicals in apples are quercetin, epicatechin, and procyanidin B2 (Lee, 2003).

Monilinia establishes infections in apple orchards, typically through wounds caused by insects or mechanically. Monilinia spp. are distributed and propagated in Asia, Europe, USA, Africa and South America. The most destructive symptom caused is apple anthracnose. Anthracnose first manifests as a small superficial, brown, circular spot on the fruit, which gradually expands. As the lesion expands, conidiophores rupture the fruit epidermis, forming small tufts. The conidiophores often form concentric rings, which radiate outwards from the initial point of infection. Soft ripe fruit, under moist conditions, with become entirely covered by conidial tufts or vegetative mycelium. Under dryer conditions, or on unripe fruits, mycelium is not present and conidial tufts are rare. Eventually affected fruits become mummified, after the whole fruit has discoloured and dehydrated. The spread of the disease to adjacent fruit occurs often, resulting in adhered clusters of mummified fruit which can persist between seasons (Byrde and Willets, 1977). 
In this study, we isolated and morphologically identified a fungal pathogen of Monilinia sp. KV-27 associated with apple anthracnose.

Isolation of fungal isolate. In this study, the rotted Fuji apples were collected from the local area of Munkyung, Kyoungbook, Republic of Korea. Isolates of anthracnose fungi were originally obtained either by direct transfer of conidia with a sterile needle from infected host tissues onto fresh potato dextrose agar (PDA) plates or by transferring infected host tissue samples into fresh $50 \mathrm{ml}$ containing potato broth (PDB) flasks after surface sterilization with $70 \%$ ethanol for $1 \mathrm{~min}$ and by washing with sterilized distilled water. The plates with conidia or infected host tissue segments were incubated at $25^{\circ} \mathrm{C}$ in darkness for 7-9 days, and hyphal tips from developing colonies, which were in most cases producing conidia of Monilinia type, were transferred to fresh PDA slants for storage and subsequent study. The culture was deposited with an accession number in the culture collection of the Laboratory of Genetic Engineering, Department of Biotechnology, Daegu University, Republic of Korea. Prior to use, fungus was grown on fresh PDA plates at $25^{\circ} \mathrm{C}$ in the dark, and small agar pieces (1-2 $\mathrm{mm}$ diameter) with mycelia were cut from the edge of the advancing colonies for inocula.

Morphology. The germination and the growth of fungus were maintained on potato dextrose agar (PDA) medium. Colony colors of the fungal isolates were described from isolates incubated at $25^{\circ} \mathrm{C}$ in dark. Morphological observations were made from structures mounted in lactophenol. The conidial measurements were derived from observations at 400x magnification (Nikon, Alphaphot-2/ YS2, China).

Isolation and morphological characterization. Exact identification of apple anthracnose pathogens (Monilinia spp.) of fruit trees should be based on laboratory studies as well as field observations of disease epidemiology and symptomatology (Ogawa et al, 1995; Van Leeuwen and Kesteren, 1998; Wormald, 1920). These studies are necessary because the fungal organs we usually find on diseased plants tissues in the field are almost always the anamorphs or conidia of the Monilinia type, which are morphologically simple and similar to each other for different species.

The fungus isolated here in this study from the rotted Fuji apples of Munkyung area of Kyounbook, Republic of Korea (Fig. 1), was identified to be Monilinia sp. KV-27
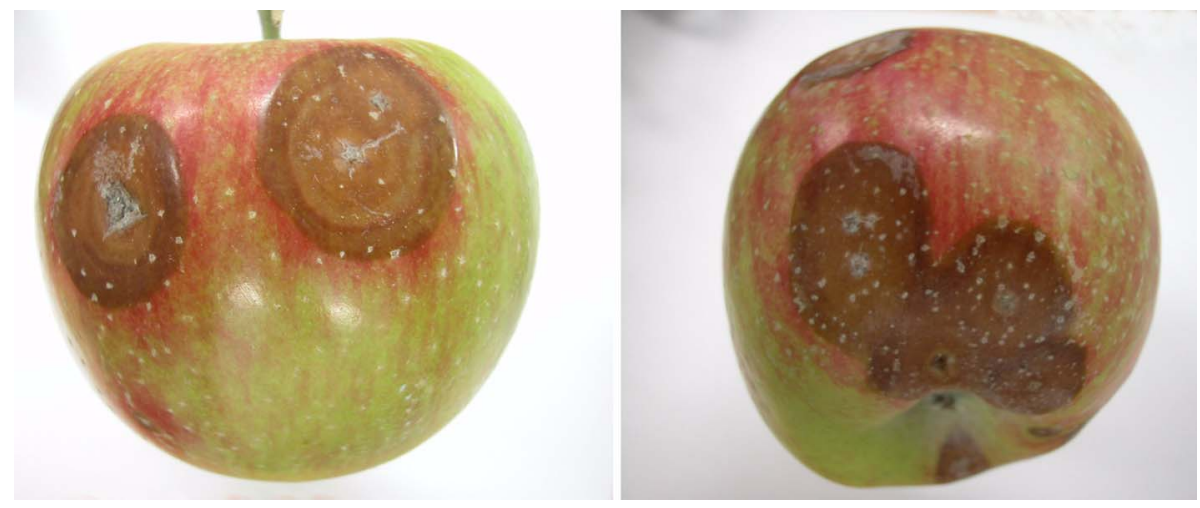

Fig. 1. Apple anthracnose of Fuji apples collected from the local area of Munkyung City, Kyoungbook, Republic of Korea.
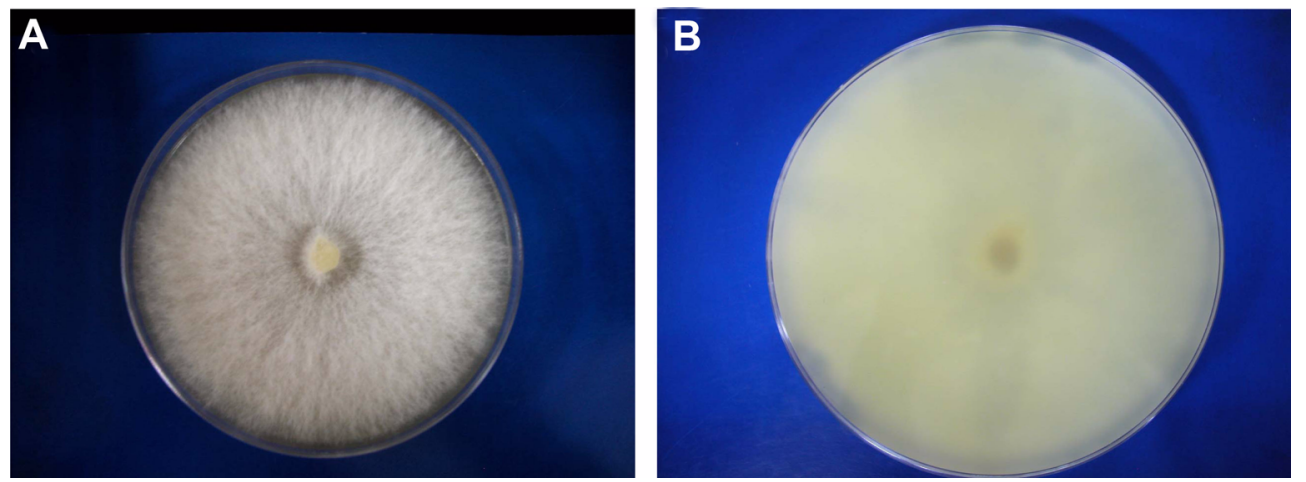

Fig. 2. Colonies of the isolate of Monilinia sp. KV-27 (front and reverse, A and B, respectively) isolated from the rotted Fuji apples. 

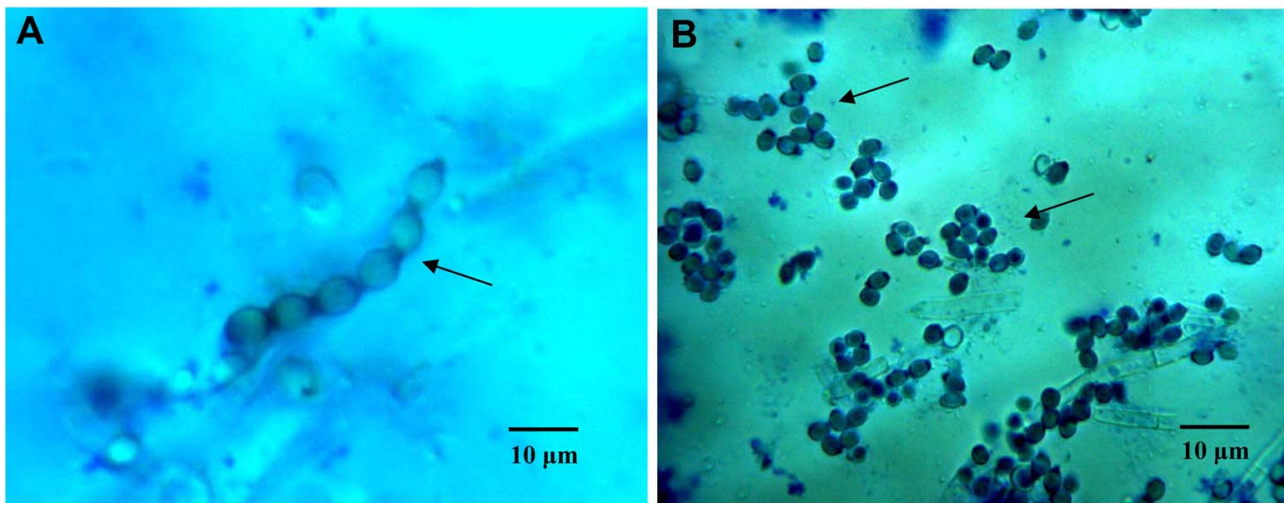

Fig. 3. Morphology of the isolate of Monilinia sp. KV-27 isolated from the rotted Fuji apples. (A) A chain of conidia of Monilinia sp. KV27; (B) Scattered conidia of Monilinia sp. KV-27.

through comparing its morphology with the documented description based on its microscopic features (Harada et al., 2004; Visarathanonth et al., 1988). As shown in Fig. 2, the colony of Monilinia sp. KV-27 grew well on fresh PDA medium and maximum mycelial growth was observed at $25^{\circ} \mathrm{C}$ with entire colony margin, filling the whole plate surface in 7 days. The colony was fluffy, grayish white, sporulating well and grayish yellow in reverse. The results of this study strongly support the identity of the fungal isolate as Monolinia sp. KV-27 and were identical to the findings of others as evident by mycelial growth and colony appearance (Harada et al., 2004; Visarathanonth et al., 1988). Aerial mycelium of Monolinia spp. at first sparse, later developing concentric zones of dense mycelium and light buff sporogenous tissue, the zonation intensified by diurnal illumination; small discoid sclerotia or irregular stromatal flakes or crusts sometimes develop on agar surface or within the medium, but in many isolates only darkening of the mycelium with age occurs (Byrde and Willetts, 1977; Hasmi et al., 1972). Primary hyphae at advancing edge of colony are thin-walled with one or more branches initiated before the first septum with dense and granular contents. Secondary and subsequent branch hyphae are often much narrower than primary hyphae. In lactophenol mounts some shrinkage of cell contents occurs and the septal pore can be seen clearly, particularly in cotton blue stain (Hasmi et al., 1972; Penrose et al., 1976).

As shown in Fig. 3, on fresh PDA, the conidia of Monilinia sp. KV-27 were blastic and formed in chains with the youngest spore at the distal end, or occasionally arthric, ellipsoid, ovoid or limoniform often with truncate ends, thin-walled, hyaline (buff-coloured in mass) which is the unique feature of genus Monilinia (Fig. 3). In tap water conidia dispersed and formed a single unbranched germ tube. Several researchers have been reported these conidial characteristics of Monilinia spp. as also evident by the findings of Visarathanonth et al., (1988).

Identification of apple anthracnose fungi which cause severe losses to pome and stone fruits including Fuji apple fruits would be an addition to plant pathology to understand the disease range of such pathogens in pre- and post-harvest technology. Thus, based on the aforementioned morphological as well as phytopathological characteristics, the isolated fungus was characterized as Monilinia sp. KV-27 However, the importance of rotted damage on Fuji apple fruits caused by Monilinia sp. KV-27 to be evaluated.

\section{Acknowledgement}

This study was supported by Technology Development Program for Agriculture and Forestry, Ministry for Agriculture, Forestry and Fisheries, Republic of Korea.

\section{References}

Apples Keep Your Family Healthy (AKYFH), 2008. Washington State Apple Advertising Commission. http://www.bestapples.com/healthy/.

Boyer, J. and Liu, R. H. 2004. Apple phytochemicals and their health benefits. Nutr. J. 3:5.

Byrde, R. J. W. and Willetts, H. J. 1977. The brown rot fungi of fruit. Pergamon Press, London, UK.

Deacon, J. W. 1997. Modern Mycology. Blackwell Scientific, Oxford, UK.

Harada, Y., Nakao, S., Sasaki, M., Sasaki, Y., Ichihashi, Y. and Sano, T. 2004. Monilia mumecola, a new brown rot fungus on Prunus mume in Japan. J. Gen. Plant Pathol. 70:297-307.

Hashmi, M. H., Morgan-Jones, G and Kendrich, B. 1972. Conidium ontogeny in Hyphomycetes: Monilia state of Neurospora sitophila and Sclerotinia laxa. Can. J. Bot. 50:2419-2421.

IAPSC, 1985. Monilinia fructicola. Distribution Maps of Major Crop Pests and Diseases in Africa No. 306. IAPSC, Yaoundé, Cameroon, South Africa. 
Lee, K. W., Kim, Y. J., Kim, D. O., Lee, H. J. and Lee, C. Y. 2003. Major phenolics in apple and their contribution to the total antioxidant capacity. J. Agric. Food Chem. 51:6516-6520.

Lee, K. W., Lee, S. J., Kang, N. J., Lee, C. Y. and Lee, H. J. 2004. Effects of phenolics in Empire apples on hydrogen peroxideinduced inhibition of gap-junctional intercellular communication. Biofactors 21:361-365.

Nutrition to Reduce Cancer Risk (NRCR), 2008. The Stanford Cancer Center (SCC). http://cancer.stanford.edu/information/ nutritionAndCancer/reduceRisk/.

Ogawa, J. M., Zehr, E. I., Bird, G. W., Ritchie, D. F., Uriu, K. and Uyemoto, J. K. 1995. Compendium of stone fruit diseases. APS, St. Paul, MN, USA.

Penrose, L. J., Tarran, J. and Wong, A. L. 1976. First record of Sclerotinia laxa in New South Wales: differentiation from $S$. fructicola by cultural characteristics and electrophoresis. Aus. J. Agric. Res. 27:547-556.

Sharma, R. 2005. Improve your health with Apple, Guava, Mango. Diamond Pocket Books (P) Ltd. pp.22. ISBN 8128809245.

Van Leeuwen, G. C. M. and van Kesteren, H. A. 1998. Delineation of the three brown rot fungi of fruit crops (Monilinia spp.) on the bases of quantitative characteristics. Can. J. Bot. 76:2042-2050.

Visarathanonth, N., Kakishima, M. and Harada, Y. 1988. Brown rot of grape berry caused by Monilinia fructicola. Ann. Phytopathol. Soc. Japan 54:238-241.

Wormald, H. 1920. The brown rot diseases of fruit trees, with special reference to two biologic forms of Monilia cinerea, Bon. II. Ann. Bot. 34:143-171. 